\title{
Crosstalk rejection in parallel optical coherence tomography using spatially incoherent illumination with partially coherent sources
}

\author{
Al-Hafeez Dhalla, ${ }^{*}$ Justin V. Migacz, and Joseph A. Izatt \\ Duke University, Department of Biomedical Engineering, Box 90281, Durham, North Carolina 27708, USA \\ *Corresponding author: al.dhalla@duke.edu
}

Received April 8, 2010; revised June 10, 2010; accepted June 10, 2010;

posted June 18, 2010 (Doc. ID 126712); published June 30, 2010

\begin{abstract}
The continuing improvement of high-speed area-scan cameras has made possible the construction of parallel optical coherence tomography (OCT) systems that are competitive with the fastest demonstrated swept-source OCT systems. Unfortunately, when imaging through turbid media using a partially coherent source, parallel OCT suffers resolution loss from coherent multiple scattering, a phenomenon known as crosstalk. We demonstrate the use of a full-field OCT system employing multimode fiber in the illumination arm to reduce the spatial coherence of a partially coherent source. By reducing the spatial coherence area below the system's lateral resolution, we create a spatial coherence gate that rejects these multiply scattered photons. We quantify the image quality and resolution improvement of this method by comparing images of a USAF test chart acquired beneath turbid phantoms using both coherent and incoherent illumination and computing the resulting modulation transfer functions. We demonstrate the feasibility of this method for imaging biological specimens by imaging a Drosophila melanogaster sample. () 2010 Optical Society of America
\end{abstract}

OCIS codes: $\quad 110.4500,110.4980,110.0113,110.2945,290.4210,290.7050$.

In recent years, the speed of optical coherence tomography (OCT) imaging systems has grown at a tremendous pace. To date, the fastest demonstrated OCT systems operate at A-scan rates of a few megahertz [1]. These high-speed systems employ a swept-source OCT (SSOCT) configuration. However, swept-source lasers capable of comparable sweep rates have not been developed in the $800 \mathrm{~nm}$ regime, which is important for ophthalmic imaging. Meanwhile, high-speed silicon-based area-scan cameras have recently achieved frame rates as high as 7000 frames per second at 1.0 megapixel resolution (Photron USA, Inc., San Diego, California). This advancement has made possible the development of high-speed parallel OCT systems in the $800 \mathrm{~nm}$ regime, using either line-field spectral domain OCT (LF-SDOCT) [2] or full-field SSOCT (FF-SSOCT) [3] configurations. Assuming 1000 spectral channels are acquired, these cameras could support A-scan rates of up to $7 \mathrm{MHz}$.

A critical concern in parallel OCT systems is crosstalk. Crosstalk originates in scattering samples when multiply scattered light reaches the detector and interferes with light originating from the coherence gated imaging depth in the sample. In point-scanning OCT, the confocal aperture of the single-mode fiber largely rejects multiply scattered light. However, parallel OCT systems must be implemented in free space and, thus, lack this confocal aperture. As a result, these methods are much more susceptible to crosstalk. Karamata et al. have provided a detailed discussion of crosstalk and the effects of multiple scattering in OCT in [4]].

Previous studies have demonstrated crosstalk rejection in parallel OCT by using spatially incoherent illumination (SII) from a thermal source [4-6]. It was demonstrated in [5] that this crosstalk rejection occurs as a result of the thermal source's low spatial coherence and is unrelated to temporal coherence. However, the average number of photons within a coherence volume of blackbody radiation is always of the order of unity
[7], which limits the speed and sensitivity of OCT systems employing thermal sources $[5,8,9]$ ]. A strategy to overcome this limit, while still achieving crosstalk rejection, is to reduce the spatial coherence of a coherent source. The theoretical basis of this approach has been developed in $[9,10]$. In this work, we demonstrate how carefully selected multimode fibers can be used to realize SII with partially coherent sources. This technique is effective in suppressing speckle and crosstalk artifacts while remaining compatible with high-speed parallel OCT.

For a step-index multimode fiber, the complex degree of mutual coherence of two points on the fiber end face at the same instant in time is given by [11]

$$
g(\Delta r)=\frac{2 \mathrm{~J}_{1}\left(k_{0} \mathrm{NA} \Delta r\right)}{k_{0} \mathrm{NA} \Delta r}
$$

where $\Delta r$ is the separation, $\mathrm{J}_{1}$ is a Bessel function of the first kind, $k_{0}$ is the wavenumber, and NA stands for the fiber NA. To quantify the effectiveness of SII to reject multiply scattered photons, we define a spatial coherence gate radius $\Delta r_{\mathrm{SCG}}$ that extends to the first minimum of the Bessel function in Eq. (1) [요, 11$]$, i.e.,

$$
\Delta r_{\mathrm{SCG}}=\frac{1.22 \pi}{k_{0} \mathrm{NA}}
$$

If the coherence gate radius of the illumination at the sample is smaller than the system resolution, the illumination can be treated as spatially incoherent [10].

Equation (1) is valid only if intermodal interactions are negligible; that is, modes propagating in the fiber must be mutually incoherent. This assumption is valid for fibers that are sufficiently long such that the intermodal delay between all supported modes exceeds the source coherence length [11]. We can meet this condition by ensuring that the intermodal delay between the fundamental mode 


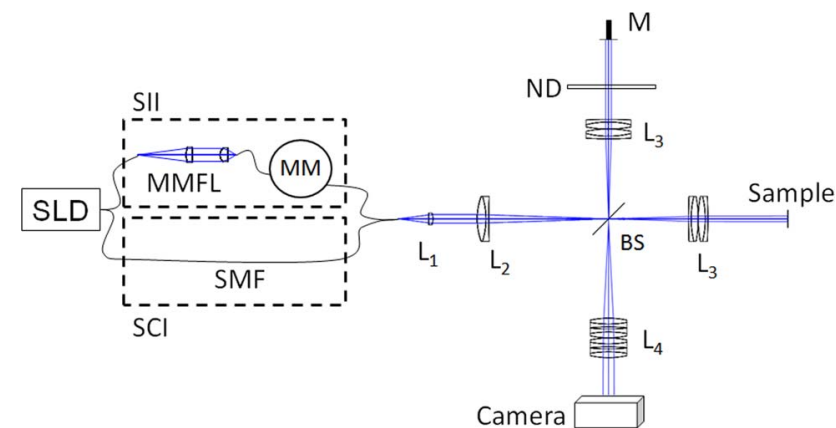

Fig. 1. (Color online) System schematic. SLD, superluminescent diode; SMF, single-mode fiber; MMFL, multimode fiber launch; MM, mode mixer and fiber spool; $\mathrm{L}_{1-4}$, lenses; $\mathrm{BS}$, beam splitter; ND, neutral density filter; M, reference mirror on PZA. Dashed boxes represent interchangeable configurations for SCI and SII.

and the highest order mode is greater than the source coherence length multiplied by the number of supported modes:

$$
\left|L_{\text {fiber }} N_{\text {high }}-L_{\text {fiber }} N_{\text {low }}\right|>l_{c} M
$$

where $L_{\text {fiber }}$ is the length of the fiber, $N_{\text {high }}$ and $N_{\text {low }}$ are the group indices of the highest order and fundamental modes, $l_{c}$ is the source coherence length, and $M$ is the number of modes supported. For a step-index multimode fiber, the difference in group index between the fundamental and highest supported modes is given by [12]

$$
N_{\text {high }}-N_{\text {low }}=N_{l} \frac{\Delta n}{n_{\text {core }}},
$$

where $N_{1}$ is the core group index, $\Delta n$ is the refractive index step between the core and cladding, and $n_{\text {core }}$ is the refractive index of the core. The number of modes supported by a weakly guiding multimode fiber with core radius $a$ is given by $M=16 a^{2}(\mathrm{NA})^{2} / \lambda^{2}$ [12]. Thus, the condition in Eq. (3) can be expressed as

$$
L_{\text {fiber }}>L_{\min }=\frac{16 a^{2}(\mathrm{NA})^{2} l_{c} n_{\text {core }}}{\lambda^{2} N_{1} \Delta n} .
$$

If this condition is met, the tip of the multimode fiber can be treated as a spatially incoherent extended source. The fiber tip can then be imaged onto the sample to realize SII, in analogy to critical illumination in microscopy. Alternatively, light emerging from the fiber can be collimated and directed to the sample, in analogy to Koehler illumination. It has been shown that both of these illumination schemes yield identical spatial coherence properties [13].

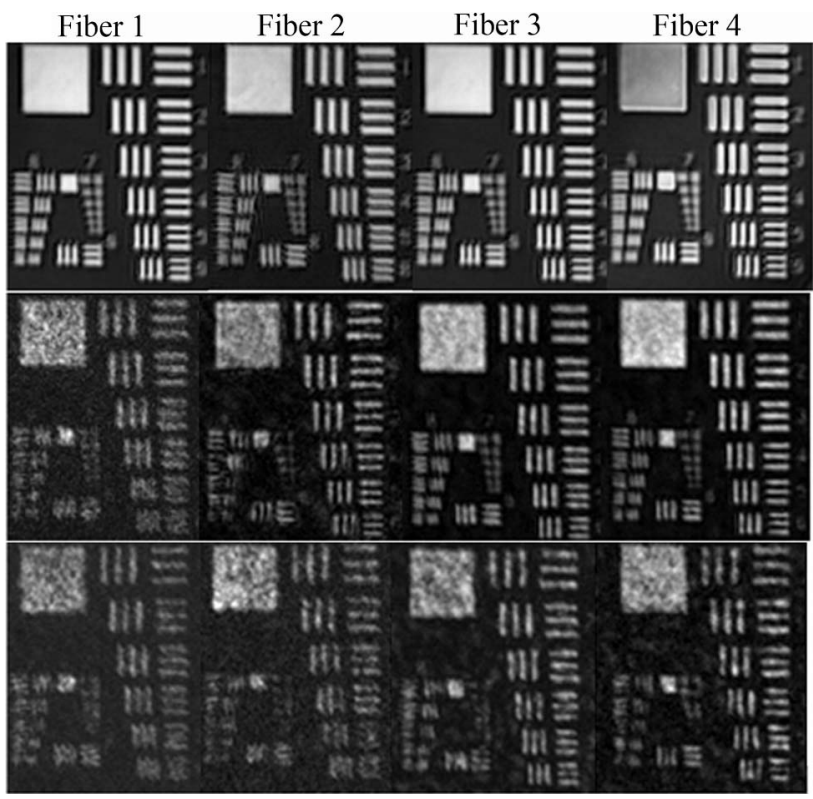

Fig. 2. USAF 1951 test chart imaged through water (top), $6 \mathrm{mfp}$ (middle), and $8 \mathrm{mfp}$ (bottom) scattering solutions.

For a proof of principle experiment, we implemented the FFOCT system depicted in Fig. 1. Major system components included a superluminescent diode [SLD, $\left.\left(\lambda_{c}=828 \mathrm{~nm}, \Delta \lambda=50 \mathrm{~nm}, P=6 \mathrm{~mW}\right)\right]$, a $1.3 \mathrm{MP}$ CMOS camera (Pixelink A741), and a reference mirror mounted on a piezoelectric actuator (PZA). The system had a field of view of $2.1 \mathrm{~mm}$ and lateral resolution of $6.9 \mu \mathrm{m}$ (Airy diameter).

Two illumination schemes were used: spatially coherent illumination (SCI) was used to demonstrate crosstalk artifacts, and SII was used to demonstrate crosstalk rejection and recovery of resolution. For SCI, a singlemode fiber was used to couple light directly from the SLD into the setup. For SII, the source was coupled into one of three multimode fibers with varying core diameters and NAs (see Table 1). For fiber 4, the coherence area is smaller than the system lateral resolution and, thus, the illumination is essentially spatially incoherent.

To resolve en face OCT images, we acquired four images in $90^{\circ}$ phase steps. Amplitude and phase maps were then extracted as described in [14]. The integration time was dynamically adjusted to account for varying coupling efficiency and sample reflectivity, and ranged from $100 \mu \mathrm{s}$ to $1 \mathrm{~ms}$. To demonstrate crosstalk rejection, we imaged a 1951 USAF resolution test chart beneath $2 \mathrm{~mm}$ solutions of water and $1 \mu \mathrm{m}$ polystyrene microspheres, containing either 0 (control), 6, or 8 mean free paths (mfp) of round-trip scattering. Using the test chart

\begin{tabular}{|c|c|c|c|c|c|c|}
\hline Fiber No. & $a$ & NA & $L_{\min }$ & $L_{\text {fiber }}$ & $M$ & $\Delta r_{\mathrm{SCG}}$ \\
\hline 1 & $8 \mu \mathrm{m}$ & 0.13 & - & $1 \mathrm{~m}$ & 1 & - \\
\hline 2 & $200 \mu \mathrm{m}$ & 0.22 & $4.4 \mathrm{~m}$ & $5 \mathrm{~m}$ & $\sim 12,500$ & $24.7 \mu \mathrm{m}$ \\
\hline 3 & $200 \mu \mathrm{m}$ & 0.48 & $3.9 \mathrm{~m}$ & $20 \mathrm{~m}$ & $\sim 53,800$ & $11.3 \mu \mathrm{m}$ \\
\hline
\end{tabular}
images, the modulation transfer function (MTF) was

Table 1. Fibers Used for Spatially Coherent and Incoherent Illumination and Relevant Parameters ${ }^{a}$

\footnotetext{
${ }^{a}$ Parameters: $a$, core diameter; NA, numerical aperture; $L_{\text {min }}$, minimum fiber length; $L_{\text {fiber }}$, actual fiber length used; $M$, number of supported modes;
} $\Delta r_{\mathrm{SCG}}$ coherence gate radius (at sample). 

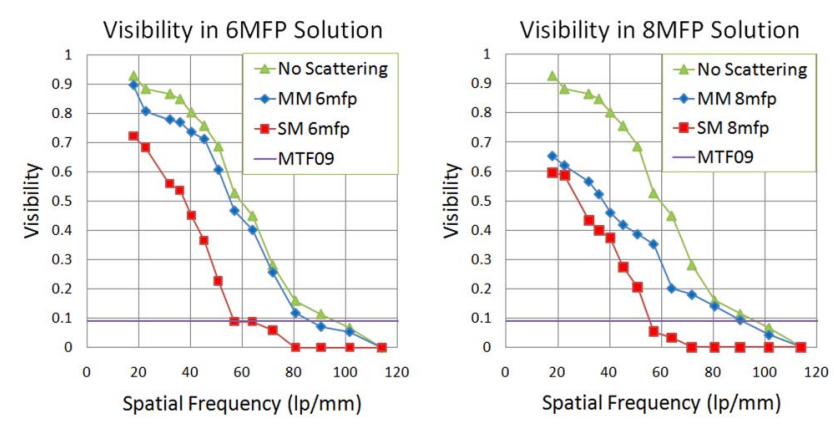

Fig. 3. (Color online) MTFs from images taken beneath water and 6 and $8 \mathrm{mfp}$ solutions for both SCI and SII.

measured for fibers 1 and 4 under all three solutions. To demonstrate the feasibility of this method for imaging biological specimens, we also imaged an immobilized Drosophila melanogaster sample using fibers 1 and 4 . Volumes were acquired by stepping the sample stage in $5 \mu \mathrm{m}$ axial steps over a range of $300 \mu \mathrm{m}$.

Images of groups 5, 6, and 7 of the test chart under all phantoms and illumination schemes are shown in Fig. 2. For the 6 and $8 \mathrm{mfp}$ solutions, a clear improvement in resolution is seen as the spatial coherence is decreased. MTF plots derived from images corresponding to fibers 1 and 4 are shown in Fig. 3. We define the effective resolution at an MTF visibility of 0.09 , as implied by the Rayleigh criterion. For the SCI case, crosstalk results in a significant loss in resolution, degrading from 95 line pairs $/ \mathrm{mm}(\mathrm{lp} / \mathrm{mm})$ in water to $60 \mathrm{lp} / \mathrm{mm}$ in the $6 \mathrm{mfp}$ solution and $55 \mathrm{lp} / \mathrm{mm}$ in the $8 \mathrm{mfp}$ solution. At high spatial frequencies, the MTF is almost completely recovered using SII, for which the resolution is $90 \mathrm{lp} / \mathrm{mm}$ for both 6 and $8 \mathrm{mfp}$. At lower spatial frequencies, the image from the $8 \mathrm{mfp}$ solution suffered some loss of contrast, likely
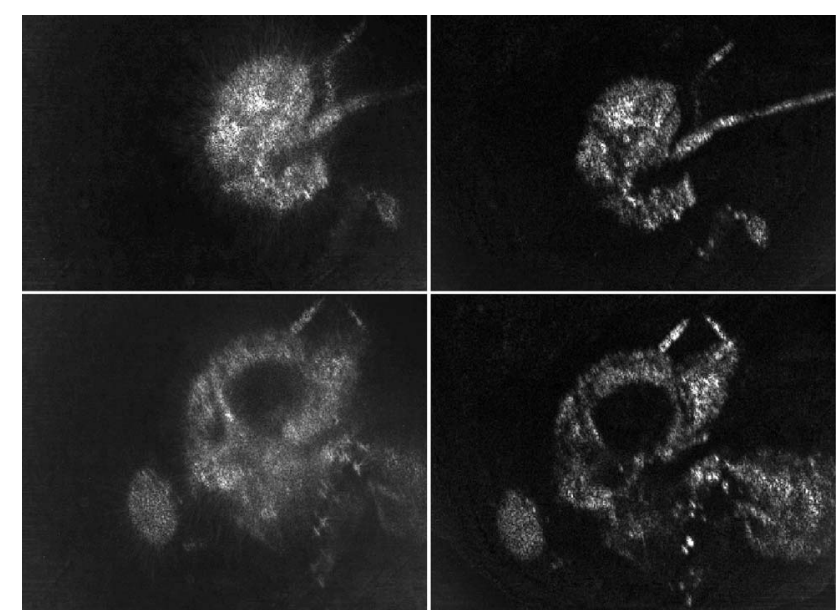

Fig. 4. OCT projections of a Drosophila melanogaster summed over $100 \mu \mathrm{m}$, centered at $100 \mu \mathrm{m}$ (top) and $200 \mu \mathrm{m}$ (bottom) from top surface using SCI (left) and SII (right). caused by the reduced signal-to-noise ratio that resulted from the additional scattering.

Representative en face projections of the fruit fly, summed over $100 \mu \mathrm{m}$, are shown in Fig. 4 . These results show degradation of image quality due to crosstalk artifacts in the SCI case, and effective rejection of these artifacts and a clear improvement in resolution in the SII case.

We have demonstrated the use of SII with a partially coherent source by employing multimode fiber, and shown that this method is effective in preventing resolution loss caused by crosstalk artifacts in turbid samples. This resolution improvement was verified quantitatively with MTF measurements, and practically by imaging a fruit fly. This technique demonstrates a solution to a major obstacle in implementing parallel acquisition OCT with partially coherent sources.

The authors thank Dr. Bing Yu and Richard Nappi for technical assistance and Dr. Nina Sherwood for providing biological specimens. We gratefully acknowledge the financial support of the U.S. Air Force Research Laboratory under contract FA8650-09-C-7932. The views, opinions, and findings contained in this letter are those of the authors and should not be interpreted as representing the official views or policies, either expressed or implied, of the Defense Advanced Research Projects Agency or the Department of Defense.

\section{References}

1. W. Wolfgang, R. B. Benjamin, K. Thomas, M. E. Christoph, and H. Robert, in OSA Technical Digest (CD) (Optical Society of America, 2010), paper BSuC1.

2. B. Grajciar, M. Pircher, A. Fercher, and R. Leitgeb, Opt. Express 13, 1131 (2005).

3. M. V. Sarunic, S. Weinberg, and J. A. Izatt, Opt. Lett. 31, 1462 (2006).

4. B. Karamata, M. Leutenegger, M. Laubscher, S. Bourquin, T. Lasser, and P. Lambelet, J. Opt. Soc. Am. A 22, 1380 (2005).

5. B. Karamata, P. Lambelet, M. Laubscher, R. P. Salathe, and T. Lasser, Opt. Lett. 29, 736 (2004).

6. L. Vabre, A. Dubois, and A. C. Boccara, Opt. Lett. 27, 530 (2002).

7. L. Mandel and E. Wolf, Optical Coherence and Quantum Optics (Cambridge Univ. Press, 1995).

8. A. F. Fercher, C. K. Hitzenberger, M. Sticker, E. Moreno-Barriuso, R. Leitgeb, W. Drexler, and H. Sattmann, Opt. Commun. 185, 57 (2000).

9. J. Kim, D. T. Miller, E. Kim, S. Oh, J. Oh, and T. E. Milner, J. Biomed. Opt. 10, 064034 (2005).

10. D. L. Marks, B. J. Davis, S. A. Boppart, and P. S. Carney, J. Opt. Soc. Am. A 26, 376 (2009).

11. S. S. Abdullaev, Sov. J. Quantum Electron. 14, 613 (1984) [Kvantovaya Elektron. 11, 904 (1984)].

12. J.-M. Liu, Photonic Devices (Cambridge Univ. Press, 2005).

13. M. Born and E. Wolf, Principles of Optics, 7th ed. (Cambridge Univ. Press, 1999).

14. G. Stoilov and T. Dragostinov, Opt. Lasers Eng. 28, 61 (1997). 